\title{
Strain overestimates non-viable myocardium in patients with ischemic mitral regurgitation: understandable discrepancy of complementary methods?
}

\author{
Jonathan Beaudoin, Mario Sénéchal \\ Institut Universitaire de Cardiologie et de Pneumologie de Québec - Université Laval, Québec, QC, Canada \\ Correspondence to: Jonathan Beaudoin, MD. Institut Universitaire de Cardiologie et de Pneumologie de Québec - Université Laval, 2725 Chemin Ste- \\ Foy, Québec, QC G1V 4G5, Canada. Email: Jonathan.beaudoin@criucpq.ulaval.ca. \\ Provenance: This is an invited Editorial commissioned by Executive Editor-in-Chief Jianxing He (Director of the Thoracic Surgery Department, The \\ First Affiliated Hospital of Guangzhou Medical University, Guangzhou, China). \\ Comment on: Morgan AE, Zhang Y, Tartibi M, et al. Ischemic Mitral Regurgitation: Abnormal Strain Overestimates Nonviable Myocardium. Ann \\ Thorac Surg 2018;105:1754-61.
}

Submitted Aug 18, 2018. Accepted for publication Sep 12, 2018.

doi: $10.21037 /$ jtd.2018.09.69

View this article at: http://dx.doi.org/10.21037/jtd.2018.09.69

Although the impact of ischemic mitral regurgitation (IMR) has been well demonstrated (1), its management remains difficult. The benefits of the surgical interventions are still controversial, and the current recommendations are subsequently much less clear than in organic MR(2). Mechanisms of IMR are related to the imbalances between the left ventricle (LV) deformation (papillary muscle displacement with subsequent valve tethering) and an inadequate mitral valve compensatory enlargement $(3,4)$. The relation between IMR and LV function is complex and two-sided: heterogeneous myocardial dysfunction (mixture of infarcted and non-infarcted segments) initially causes IMR, subsequently inducing a volume overload which in return accelerates the global LV remodeling even in noninfarcted segments. Interventions improving LV size and/ or function have therefore the potential to decrease IMR and stop this vicious cycle. After revascularization, roughly $50 \%$ of patients with IMR will improve spontaneously (without mitral valve intervention)—these odds are clinically frustrating and not helpful to guide clinical management. Predicting IMR outcome (improvement, stability, deterioration) after revascularization is critical to orient the decision to perform or not to perform additional procedures to treat IMR, mainly mitral annuloplasty or replacement. IMR spontaneous improvement after myocardial revascularization is linked to the capacity of the $L V$ to recover its function in key regions influencing mitral geometry. Greater improvements of wall motion scores after a coronary artery bypass surgery, especially in the inferior-posterior-lateral region, has been associated with a decreased IMR severity (5). Presence of viability in these myocardial segments is therefore a logical variable to analyze before a surgical or percutaneous revascularization.

The recent paper by Morgan et al. (6) explores magnetic resonance imaging (MRI) strain distribution in IMR and healthy populations. Sixteen patients with at least mild IMR and 7 healthy volunteers underwent MRI with tagging, late gadolinium enhancement (LGE) and stress perfusion. Gold standard for viability was LGE transmurality ( $<50 \%$ of wall thickness was considered viable). As expected for their selected population, the most common scar location was the inferolateral region. They found that abnormal myocardial strain can overestimate the non-viable myocardium when compared with the clinically used LGE. Presence of ischemia (using stress perfusion MRI) and infarct proximity were associated with a decreased strain in segments identified as viable by LGE. Unfortunately, no follow-up after myocardial revascularization is shown to demonstrate LV function or IMR improvement in relation with these metrics; however, this study highlights the differences between methodologies to assess myocardial viability. The results somewhat differ from previous works $(7,8)$ in which strain was found to be a good duplicate of viability obtained from a combination of SPECT, PET and LGE. Adequate 
Table 1 Concepts and methods to assess myocardial viability

\begin{tabular}{|c|c|}
\hline Methods & Indicators for hibernating myocardium \\
\hline Dobutamine stress echocardiography & - Biphasic response or worsening contractility \\
\hline \multicolumn{2}{|l|}{ Dobutamine stress MRI } \\
\hline \multicolumn{2}{|l|}{ Perfusion } \\
\hline \multicolumn{2}{|l|}{ Perfusion and metabolism } \\
\hline Single-photon emission CT & $\begin{array}{l}\text { - Initial low/absent uptake with subsequent replenishment on late } \\
\text { distribution imaging }\end{array}$ \\
\hline Positron emission tomography & - Preserved ${ }^{18}$ FDG uptake despite perfusion deficit \\
\hline \multicolumn{2}{|l|}{ Wall thickness and motion } \\
\hline Strain (echo or MRI) & - Preserved strain at baseline; increased strain with dobutamine \\
\hline \multicolumn{2}{|l|}{ Fibrosis/infarct transmurality } \\
\hline MRI late gadolinium enhancement & - Lack of transmural scarring \\
\hline
\end{tabular}

MRI, magnetic resonance imaging.

understanding of myocardial strain, viability and IMR physiology are needed to appreciate these studies as these concepts are interlinked.

\section{Myocardial viability and its potential usefulness for patients with IMR}

Myocardial viability refers to a reversible ischemic myocardial dysfunction, which can recover after revascularization. Up to now, most studies of myocardial viability have explored LV recovery, with a paucity of clinical outcome studies. In a previous study, the presence of myocardial viability by SPECT and absence of dyssynchrony by echocardiography have been associated with an improvement of IMR after revascularization (9) but overall, very few works have specifically looked at the link between viability and IMR improvement. The concept of viability is often simplified and dichotomized (myocardium is either viable or not); however, it is important to keep in mind the complexity of myocardial physiology, which can be explored with different modalities from one study to another. Viability can be assessed by multiple methods: wall thickness (10), low dose dobutamine echocardiography or MRI, thallium-201 redistribution, FDG uptake on
PET imaging or LGE on MRI (11) (Table 1). These non-invasive methods use different pathophysiological concepts to demonstrate viability, with different profiles of sensitivity and specificity. It is also important to consider the clinical setting in which viability is assessed: while non-viable myocardium reflects irreversible myocardial injury (transmural scar), the identification of a viable myocardium can reflect different physiological states which can impact IMR differently. Viable myocardium can include a healthy myocardium-not infarcted, nonischemic and with already good systolic function. This subset of viable myocardium can be identified as the normal contracting segments on a study of systolic function, and typically does not represent a clinical issue. Although noninvasive tests indicate viable myocardium, revascularization in these myocardial segments is not expected to improve IMR (already normal contractility cannot improve). Other clinically relevant types of viability are hibernating and stunned myocardium. Hibernation represents chronically ischemic, but not infarcted, myocardium which has lost its contractile properties but has the capacity to improve after revascularization. Stunned myocardium is a temporary state of myocardial dysfunction occurring after an acute ischemic event (typically already revascularized), which will 
eventually improve. Detecting hibernating and stunned myocardium in relevant myocardial segments, typically the inferoposterior wall, has the potential to give prognostic information about IMR improvement. Clinically relevant investigation should therefore be focused on identifying the ischemic segments (with anatomically related coronary artery stenosis) with decreased contractility, but with preserved viability; revascularization of these segments has the potential to improve IMR.

\section{Myocardial strain and viability}

Myocardial strain can be used to measure myocardial deformation. Global longitudinal strain is more sensitive than left ventricular ejection fraction (LVEF), to detect subtle myocardial dysfunction. Also, unlike LVEF, strain can be used regionally to assess each myocardial segment individually, which can represent an advantage to study IMR in which myocardial dysfunction is typically localized as mentioned above. Abnormal strain values, but also strain patterns such as post-systolic shortening have been associated with infarct transmurality and myocardial viability (12). Both echocardiography using speckle tracking and cardiac MRI have been used to compute myocardial strain. Strain derived from echocardiography has been used acutely after MI to predict function recovery (stunned myocardium) (13), and compared with LGE to detect infarct mass and transmurality $(14,15)$. MRI has few ways to compute myocardial strain. Myocardial tagging with or without advanced techniques [such as harmonic phase (HARP), strain encoding (SENC), displacement encoding (DENSE)] or feature tracking (16) have been described. These methods are not completely interchangeable, with different reported normal values, indicating that a work of standardization is necessary before clinical applicability. Validation of MRI tagging with invasive sonomicrometry in experimental models has nonetheless been performed with good results(17), and the strain derived from 2D tagging or SENC seems to correlate imperfectly with infarction transmurality, as defined by LGE (18-20). Most of these studies are comparing strain values in infarcted and non-infarcted myocardial segments. However, the identification of hibernating $v s$. stunned $v s$. healthy non-ischemic myocardium is not always performed and the data cannot be directly applied clinically for patients with IMR. We cannot expect viable hibernating segments to have a normal strain because strain is a measure of deformation and hibernating myocardium has abnormal contractility. It is conceivable that a transmural infarction will be associated with the most profound strain abnormalities, however, contractile properties may vary significantly in the different types of viable myocardium, from normal (or near normal) strain expected in non-ischemic segments to severely abnormal strain in hibernating segments. Finding a single cut-off strain value to define viable myocardium seems therefore problematic.

Keeping these concepts in mind, the findings of Morgan et al. are interesting and coherent. In their study, myocardial viability was defined as the absence of $>50 \%$ LGE on MRI, which therefore include healthy and hibernating (or stunned) segments. Abnormal myocardium by this LGE cut-off is therefore limited to non-viable segments. On the other hand, myocardial strain is expected to be abnormal in both the infarcted and hibernating segments (Figure 1), and even in the non-ischemic myocardium because of their selected IMR population. Although a total of 368 myocardial segments were analyzed, only those with a contractile dysfunction are really of interest to predict $L V$ and IMR improvement. The fact that strain anomalies were found in segments without transmural LGE may in fact represent an opportunity: those segments are the one with the potential to improve after revascularization. A potential clinical approach could be to identify all the segments with an abnormal strain (more sensitive approach than visual appreciation of contractility) and then evaluate their viability with either LGE or PET, while assessing viability in segments with normal strain is likely not relevant. Future studies should therefore focus on the identification of the hibernating segments. These studies will also have to include clinical and imaging outcomes after revascularization. In the clinically relevant case of predicting IMR response, the imaging should not only focus on LV recovery, but also at MR recovery.

Prediction of the IMR response to revascularization is one, among many other factors, that can be potentially helpful for guiding IMR management. Mitral valve geometry and IMR severity may help to predict the success of mitral valve repair (21-23). Another important variable to explore is the $\mathrm{LV}$ response after IMR correction since some, but not all patients will have a favorable LV reverse remodeling despite a successful IMR correction. An interesting finding in the data reported by Morgan et al. is about the globally impaired strain in IMR. This suggests a diffuse myocardial systolic impairment in these patients, and is consistent with knowledge from animal data suggesting 


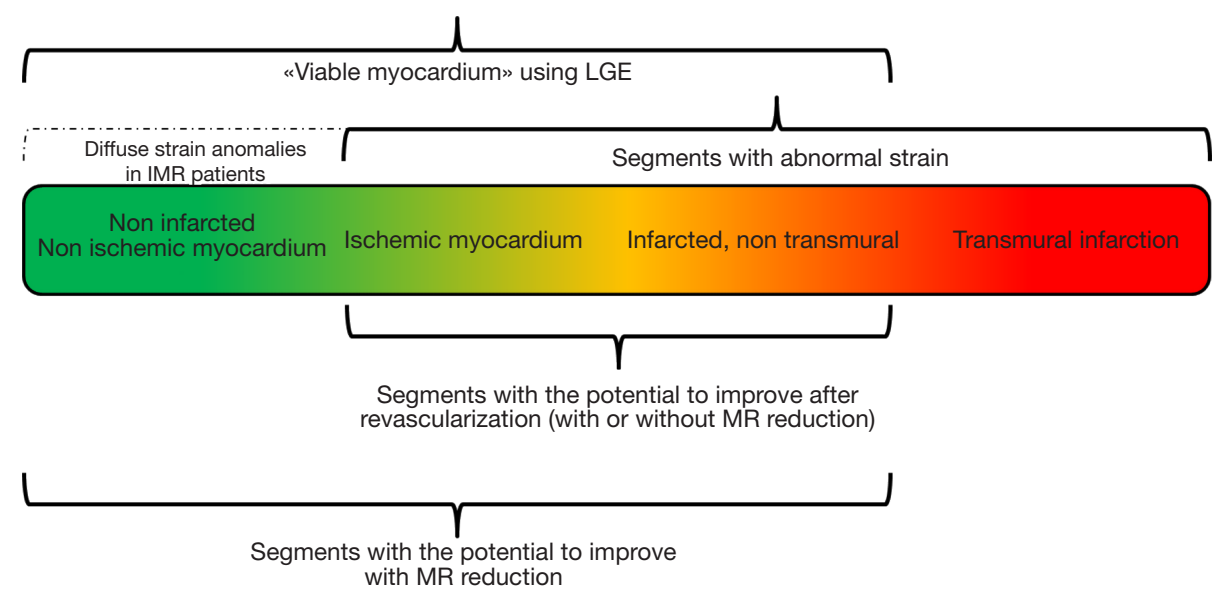

Figure 1 Spectrum of myocardial conditions encountered in patient with ischemic mitral regurgitation (IMR). Late gadolinium enhancement can detect infarct transmurality, while strain is a measure of myocardial deformation. There is an expected strain gradient from non-ischemic to transmural infarction. In patients with IMR, even non-infarcted segment have some dysfunction caused by the volume overload. Combining strain to other viability methods could potentially identify the segments most likely to improve contractility after revascularization.

whole heart changes in IMR, which are not limited to the infarcted region. Animal studies have suggested that timing is important when correcting IMR, with late correction being potentially not as useful (24). Studying the myocardial substrate of the non-infarcted myocardium could be a link to predict how IMR correction will affect global LV function. Although this was not the goal of their study, this finding by Morgan et al. hints that myocardial strain could eventually help to characterize the LV substrate and to help identify patients for which intervention for IMR is the more likely to benefit.

In conclusion, IMR is a complex disease that is difficult to manage. Prediction of its evolution after revascularization may be clinically helpful to guide the surgical decisions, and to perform or not a mitral intervention. The work presented by Morgan et al. is helpful to understand the different information that is obtained by myocardial strain and LGE. Currently, the clinical use of strain to explore viability, and to predict the IMR response after revascularization is premature. However, its integration with other modalities to explore myocardial viability will deserve future exploration. For the specific case of IMR, such studies will have to provide clinical and imaging ( $\mathrm{LV}$ function and $\mathrm{MR}$ ) outcomes based on viability and mitral geometry.

\section{Acknowledgements}

Dr. Beaudoin is funded by the Heart and Stroke Foundation of Canada and the Canadian Institutes of Health Research.

\section{Footnote}

Conflicts of Interest: The authors have no conflicts of interest to declare.

\section{References}

1. Grigioni F, Enriquez-Sarano M, Ling LH, et al. Sudden death in mitral regurgitation due to flail leaflet. J Am Coll Cardiol 1999;34:2078-85.

2. Nishimura RA, Otto CM, Bonow RO, et al. 2017 AHA/ ACC Focused Update of the 2014 AHA/ACC Guideline for the Management of Patients With Valvular Heart Disease: A Report of the American College of Cardiology/ American Heart Association Task Force on Clinical Practice Guidelines. Circulation 2017;135:e1159-95.

3. Beaudoin J, Dal-Bianco JP, Aikawa E, et al. Mitral Leaflet Changes Following Myocardial Infarction: Clinical Evidence for Maladaptive Valvular Remodeling. Circ Cardiovasc Imaging 2017;10.

4. Chaput M, Handschumacher MD, Tournoux F, et al. Mitral leaflet adaptation to ventricular remodeling: occurrence and adequacy in patients with functional mitral regurgitation. Circulation 2008;118:845-52.

5. Michler RE, Smith PK, Parides MK, et al. Two-Year Outcomes of Surgical Treatment of Moderate Ischemic 
Mitral Regurgitation. N Engl J Med 2016;374:1932-41.

6. Morgan AE, Zhang Y, Tartibi M, et al. Ischemic Mitral Regurgitation: Abnormal Strain Overestimates Nonviable Myocardium. Ann Thorac Surg 2018;105:1754-61.

7. Lancaster TS, Kar J, Cupps BP, et al. Topographic mapping of left ventricular regional contractile injury in ischemic mitral regurgitation. J Thorac Cardiovasc Surg 2017;154:149-58.e1.

8. Cupps BP, Bree DR, Wollmuth JR, et al. Myocardial viability mapping by magnetic resonance-based multiparametric systolic strain analysis. Ann Thorac Surg 2008;86:1546-53.

9. Penicka M, Linkova H, Lang O, et al. Predictors of improvement of unrepaired moderate ischemic mitral regurgitation in patients undergoing elective isolated coronary artery bypass graft surgery. Circulation 2009; 120:1474-81.

10. Cwajg JM, Cwajg E, Nagueh SF, et al. End-diastolic wall thickness as a predictor of recovery of function in myocardial hibernation: relation to rest-redistribution T1201 tomography and dobutamine stress echocardiography. J Am Coll Cardiol 2000;35:1152-61.

11. Kim RJ, Wu E, Rafael A, et al. The use of contrastenhanced magnetic resonance imaging to identify reversible myocardial dysfunction. N Engl J Med 2000;343:1445-53.

12. Eek C, Grenne B, Brunvand H, et al. Postsystolic shortening is a strong predictor of recovery of systolic function in patients with non-ST-elevation myocardial infarction. Eur J Echocardiogr 2011;12:483-9.

13. Mollema SA, Delgado V, Bertini M, et al. Viability assessment with global left ventricular longitudinal strain predicts recovery of left ventricular function after acute myocardial infarction. Circ Cardiovasc Imaging 2010;3:15-23.

14. Chan J, Hanekom L, Wong C, et al. Differentiation of subendocardial and transmural infarction using twodimensional strain rate imaging to assess short-axis and long-axis myocardial function. J Am Coll Cardiol 2006;48:2026-33.

15. Gjesdal O, Helle-Valle T, Hopp E, et al. Noninvasive separation of large, medium, and small myocardial infarcts in survivors of reperfused ST-elevation myocardial infarction: a comprehensive tissue Doppler and speckletracking echocardiography study. Circ Cardiovasc Imaging 2008;1:189-96, 2 p following 196.

16. Vo HQ, Marwick TH, Negishi K. MRI-Derived
Myocardial Strain Measures in Normal Subjects. JACC

Cardiovasc Imaging 2018;11:196-205.

17. Yeon SB, Reichek N, Tallant BA, et al. Validation of in vivo myocardial strain measurement by magnetic resonance tagging with sonomicrometry. J Am Coll Cardiol 2001;38:555-61.

18. Altiok E, Neizel M, Tiemann S, et al. Layer-specific analysis of myocardial deformation for assessment of infarct transmurality: comparison of strain-encoded cardiovascular magnetic resonance with 2D speckle tracking echocardiography. Eur Heart J Cardiovasc Imaging 2013;14:570-8.

19. Oyama-Manabe N, Ishimori N, Sugimori H, et al. Identification and further differentiation of subendocardial and transmural myocardial infarction by fast strainencoded (SENC) magnetic resonance imaging at 3.0 Tesla. Eur Radiol 2011;21:2362-8.

20. Amzulescu MS, Langet H, Saloux E, et al. Head-to-Head Comparison of Global and Regional Two-Dimensional Speckle Tracking Strain Versus Cardiac Magnetic Resonance Tagging in a Multicenter Validation Study. Circ Cardiovasc Imaging 2017;10.

21. Magne J, Senechal M, Mathieu P, et al. Restrictive annuloplasty for ischemic mitral regurgitation may induce functional mitral stenosis. J Am Coll Cardiol 2008;51:1692-701.

22. Magne J, Pibarot P, Dagenais F, et al. Preoperative posterior leaflet angle accurately predicts outcome after restrictive mitral valve annuloplasty for ischemic mitral regurgitation. Circulation 2007;115:782-91.

23. Goldstein D, Moskowitz AJ, Gelijns AC, et al. Two-Year Outcomes of Surgical Treatment of Severe Ischemic Mitral Regurgitation. N Engl J Med 2016;374:344-53.

24. Beaudoin J, Levine RA, Guerrero JL, et al. Late repair of ischemic mitral regurgitation does not prevent left ventricular remodeling: importance of timing for beneficial repair. Circulation 2013;128:S248-52.

(English Language Editor: Jeremy Dean Chapnick, AME Publishing Company)

Cite this article as: Beaudoin J, Sénéchal M. Strain overestimates non-viable myocardium in patients with ischemic mitral regurgitation: understandable discrepancy of complementary methods? J Thorac Dis 2018;10(Suppl 33):S3946-S3950. doi: 10.21037/jtd.2018.09.69 